\title{
Resultados de aprendizaje en las evaluaciones censales de los estudiantes del segundo grado de primaria de las instituciones educativas unidocentes multigrado de la región Cusco (Perú) ${ }^{1}$
}

\author{
Luis Zúniga Alvarez²
}

Recibido 04 de noviembre 2018 - Aceptado 29 de noviembre 2018

\section{RESUMEN}

El presente artículo describe los logros de aprendizaje obtenidos por los estudiantes del segundo grado de primaria de las instituciones educativas unidocentes multigrado (IIEEUM) de la región Cusco, Perú, en las evaluaciones censales que anualmente implementa el Ministerio de Educación. Conocer estos resultados permite comprobar el cumplimiento del derecho a la educación en el sistema educativo peruano. Dichas evaluaciones censales dan cuenta de que, en general, los logros de aprendizaje en comprensión lectora y matemática obtenidos por niños y niñas de las instituciones educativas del nivel primario de las zonas rurales se sitúan en los niveles más bajos. No existen estudios sobre los logros de aprendizaje en las instituciones educativas unidocentes multigrado. Desde los resultados de este estudio cuantitativo, se concluye que son pocos los niños y niñas de estas instituciones educativas que logran alcanzar el nivel satisfactorio. Si se comparan estos resultados a nivel de cada Unidad de Gestión Educativa Local (UGEL) no existen diferencias significativas y el efecto de estos resultados en el porcentaje regional es mínimo, situación que invita a investigadores, especialistas y decisores en política educativa a enfocar su interés en la complejidad de la realidad educativa en el medio rural.

Palabras clave: logros de aprendizaje, instituciones educativas unidocentes multigrado, zona rural, evaluación censal.

I El presente artículo forma parte del trabajo de investigación intitulado: "Efecto de los resultados de aprendizaje obtenidos en las evaluaciones censales por los estudiantes del segundo grado de primaria de las instituciones educativas unidocentes multigrado de la región Cusco, en el puntaje regional" que se presentó en el VI Seminario Nacional de Investigación Educativa y III Coloquio Iberoamericano de Educación Rural, realizado del 8 al 10 de noviembre de 2018, en la región Cusco, Perú, organizado por la Sociedad de Investigación Educativa Peruana (SIEP) y la Red Iberoamericana de Educación Rural.

2 Magister en Ciencias de la Educación con mención Administración Educacional de la Pontificia Universidad Católica de Chile. Docente de la Quinta Escala Magisterial en la IE San Francisco de Borja Cusco. E mail: lizuniga@uc.cl 


\title{
Learning outcomes in the census evaluations of second grade students in elementary school in multigrade single-teacher educational institutions of Cusco region (Peru)
}

\begin{abstract}
This article describes the learning achievements obtained by second grade students in elementary school at multigrade educational institutions of Cusco region, Peru, in the annual census evaluations implemented by the Ministry of Education. With these results it is possible to verify the fulfillment of the right to education in the Peruvian system. In general, these census evaluations show very low levels of learning achievements in reading comprehension and mathematics obtained by children from primary education institutions in rural areas. There are no studies on learning achievements in multigrade single-unit educational institutions. Some results of this quantitative study conclude that few children from these educational institutions manage to reach a satisfactory level. If these results are compared among every UGEL (local education management unit), there are no significant differences and the effect of these results on the regional percentage is minimal. This situation invites researchers, specialists and decision-makers in educational policy to focus their interest on the complexity of the reality of education in rural areas.
\end{abstract}

Key words: Learning achievements, multigrade single-unit educational institutions, rural area, census evaluation.

\section{Introducción}

Mejorar los aprendizajes de todos los niños y niñas constituye el objetivo central de toda política educativa (Fumagalli, 20I5). En este sentido, muchos sistemas educativos en el mundo, incluido el sistema educativo peruano, vienen adoptando diferentes medidas tendientes a mejorar los niveles de aprendizaje obtenidos por los estudiantes de la educación básica regular.

Los resultados de las evaluaciones, nacionales e internacionales, que implementa el Ministerio de Educación informan que los estudiantes del segundo grado de primaria, en su gran mayoría, no alcanzan los niveles más altos en comprensión lectora y matemática (Miranda, 2008). Los niños y niñas de las zonas rurales tienen los más bajos puntajes en relación a sus pares de las zonas urbanas (UMC Minedu, 2005; CNE, 2016) No existen estudios sobre los resultados que obtienen los educandos de las instituciones educativas unidocentes multigrado que se localizan en los lugares más alejados de las zonas rurales de la región Cusco. 
Este artículo parte de un estudio realizado en la región Cusco acerca de los resultados de aprendizaje obtenidos por los estudiantes del segundo grado de primaria de las instituciones educativas unidocentes multigrado (IIEEUM) de la región Cusco, en las evaluaciones censales de los años 20I4, 2015 y 20I6. El objetivo general de dicho estudio fue describir los resultados de aprendizaje de los estudiantes del segundo grado de primaria, de las IIEEUM, que se encuentran en el nivel satisfactorio en comprensión lectora y capacidades matemáticas, y su efecto en el porcentaje total de la región Cusco. Para ello se realizó, primero, una descripción general de los resultados de cada Unidad de Gestión Educativa Local, UGEL, sobre el porcentaje de estudiantes del segundo grado de primaria de las IIEEUM que lograron el Nivel Satisfactorio (NS) en comprensión lectora y capacidades matemáticas. Posteriormente se identificó aquellas UGEL que tienen el mayor porcentaje de estudiantes del segundo grado de primaria de las IIEEUM, en el NS, y finalmente se determinó el efecto del porcentaje total de estudiantes del segundo grado de primaria de las IIEEUM que alcanzaron el NS en el porcentaje general de la región Cusco.

Para una mejor comprensión de la problemática antes descrita, este artículo inicia con un análisis del sentido y alcances del derecho a la educación en la legislación educativa del Perú. La Ley General de Educación $N^{\circ} 28044$, en diferentes capítulos, enfatiza la obligación de brindar las facilidades necesarias para que todos los estudiantes accedan a un servicio educativo de calidad, garantizar su trayectoria educativa y asegurarles aprendizajes con calidad y equidad.

Enseguida, propone una breve caracterización de las condiciones y limitaciones de las instituciones educativas unidocentes multigrado con las cuales brindan el servicio educativo en las zonas rurales, entre estas: la ubicación geográfica, la infraestructura y el equipamiento con que cuentan, el acceso a los servicios básicos, los roles que asume el profesorado, las características de los estudiantes y el tiempo efectivo para los procesos de enseñanza-aprendizaje (Ames, 2004). Estas condiciones influyen en el trabajo pedagógico en las aulas multigrado, que se constituyen también en potenciales espacios para el desarrollo cognitivo y socioemocional de los niños y niñas del medio rural. 
Otro tópico a tratar corresponde a la evolución de las instituciones educativas unidocentes multigrado en la región Cusco. Se advierte un notable incremento de estas en los últimos años, aun cuando, contrariamente, su matrícula escolar viene decreciendo, fenómeno que muestra una atomización de los servicios educativos en las zonas rurales y que podría deberse a las condiciones de una nueva ruralidad en el país.

Concluye con la presentación de los resultados, que dan cuenta que es mínimo el porcentaje de niños y niñas de las instituciones educativas unidocentes multigrado de la región Cusco que se ubican en el nivel satisfactorio en comprensión lectora y matemática. No existen diferencias significativas al momento de agrupar dichos porcentajes en términos de las unidades de gestión educativa local a la cual pertenecen y, finalmente, este porcentaje de estudiantes que alcanzan el nivel satisfactorio no afecta significativamente en el porcentaje total regional.

\section{Las instituciones educativas unidocente multigrado y el derecho a la educación en el sistema educativo del Perú}

El Derecho a la Educación (DE), constituye un derecho humano, reconocido mundialmente, que se encuentra establecido en la Declaración Universal de los Derechos Humanos, los Pactos Internacionales, la Convención de los Derechos del Niño y otros tratados y declaraciones internacionales, como la Declaración Mundial de Educación para todos, entre otros.

La Convención de los Derechos del Niño, suscrita en I989, en el Artículo 28, numeral I, se refiere a la importancia de garantizar la obligatoriedad y gratuidad de la enseñanza de la educación primaria para todos, y de las medidas necesarias a adoptar para asegurar el acceso y la permanencia de todos los niños y niñas. Ello significa que todo Estado debe asegurar las condiciones necesarias para que todas las personas, sin importar su origen y condición social, puedan culminar exitosamente la trayectoria educativa establecida por el sistema educativo y, por consiguiente, alcanzar su desarrollo integral. 
Entre el DE y los derechos humanos existe una relación intrínseca, por cuanto constituye el nexo entre todos los demás derechos universales. En este sentido, Latapí (2009) afirma que:

Los especialistas en derechos humanos resaltan la relación del DE con los demás derechos humanos. La educación potencia el desarrollo de la persona y, por ello, es condición esencial para el disfrute de todos ellos; por esto se considera éste como un derecho clave (key right). No se puede ejercer ninguno de los derechos civiles, políticos, sociales, económicos o culturales sin un mínimo de educación. Por ejemplo: la libertad de expresión: ¿de qué sirve si la persona no tiene las capacidades de formarse un juicio personal y de comunicarlo? (p. 258).

Inicialmente, el derecho a la educación fue entendido como el derecho de las personas a recibir educación. Esta visión permitió garantizar la expansión y la cobertura de los servicios educativos para toda la población. Sin embargo, dadas las situaciones de inequidad educativa, que se reflejan en los índices de repitencia, deserción y abandono escolar en muchos sistemas educativos, especialmente en los países en vías de desarrollo, se considera que el DE, además de garantizar la permanencia de los estudiantes y la culminación de las trayectorias educativas establecidas, también debe asegurar que todos los estudiantes alcancen los aprendizajes establecidos en el currículo escolar (Fumagalli, 20I5; Vigo \& Nakano, 2007).

En el Perú, la Ley General de Educación N² 28044, en el TÍTULO I Fundamentos y disposiciones generales, en el Artículo $8^{\circ}$. Principios de la educación, pone de relieve a la persona como centro y agente fundamental del proceso educativo y, para garantizar el pleno ejercicio de este derecho, establece los principios de equidad, inclusión, calidad, entre otros.

En la misma Ley, el CAPÍTULO IV La equidad en la educación, Artículo $17^{\circ}$. Equidad en la educación, literalmente señala que:

Para compensar las desigualdades derivadas de factores económicos, geográficos, sociales o de cualquier otra índole que afectan la igualdad de oportunidades en el ejercicio del derecho a la educación, el Estado toma medidas que favorecen 
a segmentos sociales que están en situación de abandono o de riesgo para atenderlos preferentemente. (p.IO).

Siendo esto así, también todos los niños y niñas que estudian en las IIEEUM deberían tener las condiciones necesarias para alcanzar mejores aprendizajes; sin embargo, el cumplimiento del DE enfrenta una diversidad de obstáculos y retos en cuanto a la calidad de los servicios educativos que reciben, tales como: la falta de dotación oportuna de docentes, inadecuada infraestructura y equipamiento para el desarrollo de los procesos de enseñanza-aprendizaje, docentes que ignoran la lengua materna de los educandos, solo por citar algunos (Ames, 2004).

\section{Definición y caracterización de las IIEEUM}

Las IIEEUM, llamadas también "escuelas multiedad”, "escuelas unitarias", "escuelas de maestro único" o "de clases múltiples", son aquellas que reúnen a niños y niñas de diferentes edades y grados en una sola aula, por lo general a cargo de un docente, y se ubican habitualmente en las zonas rurales más alejadas de la costa, sierra y selva, en zonas escasamente pobladas y/o donde la población se halla dispersa. A estas concurren hablantes de un idioma materno, que puede ser el castellano, quechua, aymara, o alguna lengua amazónica, según sea el espacio geográfico en que se localicen.

El servicio educativo en las IIEEUM se presenta de diversas formas (Ames, 2004). En unos casos es posible encontrar a un docente atendiendo cuatro o seis grados de educación primaria; en otros, un docente trabajando con dos grados a la vez o, también dos docentes que se reparten tres grados cada uno o los dos primeros grados uno y los cuatro restantes el otro.

En la actualidad, otros sistemas educativos consideran a las escuelas multigrado como una alternativa pedagógica que busca potenciar los procesos pedagógicos mediante el desarrollo de las interacciones entre los educandos de distintas edades en el aula.

Una primera característica que resalta de las IIEEUM es la ubicación geográfica. Estas se localizan en los lugares más apartados 
e inaccesibles de las zonas rurales, situación que condiciona todo tipo de atención por parte del Estado y dificulta (dependiendo de la distancia y de acceso vial) la llegada oportuna de los profesores.

Las prácticas pedagógicas se desarrollan, generalmente, en ambientes precarios y con un escaso equipamiento, situación que repercute en el normal desarrollo de los procesos de enseñanza-aprendizaje (IPEBA, 20I I). Además, una gran parte de las IIEEUM no cuentan con acceso a los servicios básicos -agua potable, desagüe y servicio de energía eléctrica (CNE, 2016)-.

El docente que se desempeña en estas instituciones educativas trabaja con un número mínimo de estudiantes. Aun cuando la Resolución de la Secretaría General N ${ }^{\circ}$ I825-20I4-Minedu establece que la ratio (proporción) de estudiantes por docente en el aula multigrado rural y unidocente es de veinte estudiantes, la proporción real de estudiantes por docente es de trece estudiantes (Escale, 2017). A la situación antes descrita hay que agregar que un buen número de docentes que laboran en las IIEEUM todavía no manejan el idioma de los estudiantes, otros no cuentan con formación profesional en pedagogía y los que la tienen no están debidamente formados para trabajar con aulas heterogéneas. También, la permanencia de muchos profesores es corta, por cuanto la expectativa de muchos de ellos es siempre conseguir una plaza en las capitales distritales (Ames, 2004).

Otro aspecto importante es el hecho de que el profesor en las IIEEUM asume un doble rol, porque, además, es el director de la institución educativa. El profesor-director debe compartir la jornada laboral en el ejercicio de ambos roles y, por lo mismo, se ve obligado a suspender las sesiones de clase para atender diversas cuestiones administrativas, hecho que afecta negativamente el aprendizaje de los estudiantes y el cumplimiento de los compromisos de gestión escolar establecidos por el Ministerio de Educación (Minedu).

Los niños y niñas que estudian en las IIEEUM, dependiendo de la zona geográfica en que viven (costa, sierra o selva), son hablantes de una lengua materna. Además, son poseedores de una cultura originaria, un sistema de valores, unas tradiciones, costumbres y creencias que les permiten interpretar y explicar la particularidad 
de ese espacio y reconocerlo como parte de su identidad. Estas características son potencialmente aprovechadas en la planificación y ejecución de los procesos pedagógicos.

Las familias de estos niños y niñas viven en una situación socioeconómica de extrema pobreza (IPEBA, 20I I; CNE, 20I6). Esta situación repercute en la calidad de la alimentación y en la salud de estudiantes, y por ende en el rendimiento escolar de estos (León \& Collahua, 2016). Además, los niños y niñas ayudan en las actividades económicas de la familia, generalmente agrícolas; por lo mismo, hay temporadas en las cuales dejan de asistir a clases, debido a la demanda de mano de obra en la familia.

En cuanto al tiempo para el aprendizaje, en las IIEEUM este se reduce a tres o cuatro días, debido a que la institución educativa se encuentra en un lugar distante que hace que el desplazamiento de los docentes tome mucho tiempo, entre otras situaciones; además, los horarios son irregulares debido a la atención de funciones no pedagógicas que desempeña el profesor (IPEBA, 20II). Las realidades antes descritas explican que el promedio de horas que se destine para los aprendizajes es significativamente menor.

Estas y otras características definen la identidad compleja de las IIEEUM y requieren ser ampliamente estudiadas; por ello, este trabajo de investigación invita a seguir explorando la realidad de la educación en las zonas más alejadas del ámbito rural y aportar a su conocimiento; de este modo, contribuir a su mejor comprensión y atención por parte de los decisores en políticas educativas.

\section{El aula multigrado: procesos pedagógicos y potencialidades para su desarrollo}

Las practicas pedagógicas que realizan los profesores en el aula multigrado (en solitario) es altamente compleja y desafiante, puesto que estos docentes no han tenido la formación docente inicial necesaria para gestionar los aprendizajes en aulas multigrado. Además, los programas de acompañamiento docente, en general, no llegan a la totalidad de docentes de estas instituciones educativas. 
Trabajar la heterogeneidad en las aulas multigrado exige de una formación inicial docente (también de una formación continua) especializada, considerando que estas aulas acogen a niños y niñas de diferentes edades y niveles de desarrollo cognitivo y psicosocial, y esta diversidad exige de una cuidadosa planificación de los procesos de evaluación, de la enseñanza y el aprendizaje (Anijovich, 20I4; Vargas, 2003).

La enseñanza personalizada caracteriza a los procesos pedagógicos en las IIEEUM. Dado que, en el aula multigrado, la cantidad de estudiantes es menor, la fluidez de las interacciones comunicativas entre profesor-estudiante y estudiante-estudiante se ve favorecida; además, el profesor puede conocer mejor las características, necesidades y potencialidades de cada uno de los niños y niñas. Esta enseñanza individualizada puede complementarse mejor con el trabajo cooperativo o en equipos de estudiantes.

Tal forma de organización para el aprendizaje en el aula multigrado puede contribuir a la configuración de un clima de trabajo agradable para el aprendizaje en el aula e incidir positivamente en el desarrollo socioemocional de los estudiantes. Existe evidencia empírica que señala que el profesor se constituye en una figura de apego secundario en el aula, es decir, asume el rol de mediador socioemocional. En este sentido, Berger, Álamos y Milicic (2016), citando a Pianta et al. (2012); Sabol y Pianta (2012) afirman que:

Este patrón de interacción, caracterizado por la sensibilidad y responsividad del adulto, reflejaría una cierta confianza de los estudiantes en el profesor, la cual se manifiesta, por ejemplo, en que los alumnos participan en las clases por iniciativa propia, piden ayuda si es necesario y se atreven a cometer errores, entre otros. (p. 390).

En consecuencia, el aula multigrado constituye un espacio ecológico privilegiado para el desarrollo cognitivo y socioemocional de los niños y niñas que atiende, es por ello que el desempeño del profesor es determinante en este espacio escolar. Al respecto, Anijovich (20I4) manifiesta que: 
Para trabajar con este enfoque necesitamos contar con docentes que tengan lo que llamamos una "mentalidad de crecimiento", es decir, que vean en los alumnos un mundo de posibilidades $[\ldots]$ Es importante que conozcan bien su rol a la hora de establecer altos objetivos y proveer un sólido soporte, como también estar en la búsqueda de lo que funciona para que sus estudiantes alcancen el máximo potencial en la escuela, aun en situaciones complejas. (p. 28).

Atender esta exigencia requiere de una formación inicial y continua del profesorado en general, asumiendo transversalmente el enfoque de la heterogeneidad en los procesos de enseñanza-aprendizaje. De esta manera, también se podrá contribuir de manera efectiva a un buen desempeño del profesorado que labora en las IIEEUM y cuyos desempeños se encuentran ampliamente descritos en el Marco de Buen Desempeño Docente (20I2).

\section{Las IIEEUM en el contexto de la educación primaria en la región Cusco}

La educación primaria en el Perú constituye el segundo nivel de la educación básica regular, que atiende a niños y niñas en edad escolar a partir de los seis años de edad y tiene una duración de seis años, conforme establece la normativa oficial de nuestro sistema educativo (Ley General de Educación 28.044).

Actualmente, a nivel nacional existen 29.360 instituciones educativas en el Nivel Primario, de estas, 22.I I 2 se encuentran ubicadas en las diversas zonas rurales de nuestras regiones. La región Cusco cuenta con 1.606 instituciones educativas entre las zonas rural y urbana (Escale, 2017).

El servicio educativo en el nivel primario se ofrece mediante instituciones educativas que adoptan formas o características diferentes: instituciones educativas unidocentes multigrado, instituciones educativas polidocentes multigrado e instituciones educativas polidocentes completos. La tabla I muestra la evolución del servicio educativo en estas modalidades en los niveles nacional y regional. 
Tabla I. IIEE unidocentes multigrado, polidocentes multigrado y polidocentes completos.

\begin{tabular}{|l|r|r|r|r|r|r|}
\hline IIEE del Nivel & \multicolumn{2}{|c|}{2015} & \multicolumn{2}{|c|}{2016} & \multicolumn{2}{|c|}{2017} \\
\cline { 2 - 7 } $\begin{array}{l}\text { Primario por el } \\
\text { tipo de servicio }\end{array}$ & Nacional & $\begin{array}{l}\text { Región } \\
\text { Cusco }\end{array}$ & Nacional & $\begin{array}{l}\text { Región } \\
\text { Cusco }\end{array}$ & Nacional & $\begin{array}{l}\text { Región } \\
\text { Cusco }\end{array}$ \\
\hline $\begin{array}{l}\text { Unidocentes } \\
\text { multigrado }\end{array}$ & 8.669 & 310 & 8.895 & 329 & 8.212 & 345 \\
\hline $\begin{array}{l}\text { Polidocentes } \\
\text { multigrado }\end{array}$ & 11.429 & 794 & 11.365 & 778 & 11.382 & 762 \\
\hline $\begin{array}{l}\text { Polidocentes } \\
\text { completos }\end{array}$ & 1.918 & 180 & 1.812 & 179 & 1.766 & 170 \\
\hline
\end{tabular}

Fuente: Elaboración propia con datos del ESCALE - MINEDU.

En la tabla I se aprecia que, en la región Cusco, en los tres últimos años, la cantidad de IIEEUM se ha venido incrementando a la par que las polidocentes completas, $y$ las polidocentes multigrado han venido disminuyendo. En el presente año 2018 y con aprobación del Minedu, mediante la R.D. N I89-20I8-ED, se cuenta con 393 IIEEUM.

En cuanto a la matrícula escolar, de acuerdo al Censo Escolar de los años 2015, 2016 y 2017, se puede constatar que la población escolar en las IIEEUM en la región Cusco viene decreciendo, tal y conforme se puede apreciar en la tabla 2:

Tabla 2. Matrícula escolar en IIEE unidocentes multigrado, polidocentes completos.

\begin{tabular}{|l|r|r|r|r|r|r|}
\hline IIEE del Nivel & \multicolumn{2}{|c|}{2015} & \multicolumn{2}{c|}{2016} & \multicolumn{2}{c|}{2017} \\
\cline { 2 - 7 } $\begin{array}{l}\text { Primario por el } \\
\text { tipo de servicio }\end{array}$ & Nacional & $\begin{array}{l}\text { Región } \\
\text { Cusco }\end{array}$ & Nacional & $\begin{array}{c}\text { Región } \\
\text { Cusco }\end{array}$ & Nacional & $\begin{array}{c}\text { Región } \\
\text { Cusco }\end{array}$ \\
\hline $\begin{array}{l}\text { Unidocentes } \\
\text { multigrado }\end{array}$ & $\mathrm{I} 29.740$ & 4.455 & $\mathrm{I} 23.82 \mathrm{I}$ & 4.204 & $\mathrm{I} 37.582$ & 4.033 \\
\hline $\begin{array}{l}\text { Polidocentes } \\
\text { completos }\end{array}$ & $\mathrm{I} 29.347$ & $\mathrm{I} 7.364$ & $\mathrm{I} 40.055$ & $\mathrm{I} 7.704$ & $\mathrm{I} 32.379$ & $15.95 \mathrm{I}$ \\
\hline
\end{tabular}

Fuente: Elaboración propia con datos del ESCALE - MINEDU.

Si se compara la cantidad de IIEEUM con la cantidad de estudiantes matriculados durante 2015, 2016 y 2017, se puede advertir que, la cantidad de IIEEUM viene aumentando en la región Cusco, mientras que la matrícula escolar disminuye, situación que obedece a una tendencia a la atomización de los programas y servicios educativos, con bajo número de matriculados y con aulas con un número reducido de estudiantes (Guadalupe, León, Rodríguez \& Vargas, 2017). 


\section{Resultados de la Evaluación Censal de Estudiantes (ECE) en la educación primaria}

Todas las instituciones educativas de la educación básica regular tienen el objetivo de garantizar un servicio educativo con calidad y equidad para los estudiantes, según establece el derecho a la educación y conforme también se plantea en el Proyecto Educativo Nacional (2007), en el Objetivo Estratégico I Oportunidades y resultados educativos de igual calidad para todos y, Objetivo Estratégico 2, Estudiantes e instituciones que logran aprendizajes pertinentes $y$ de calidad. Sin embargo, los resultados de aprendizaje de niños y niñas en las zonas rurales demuestran la persistencia de notorias disparidades en los logros de aprendizajes.

A partir de 2007, el Minedu, a través de la Oficina de Medición de la Calidad de los Aprendizajes, implementó la Evaluación Censal de Estudiantes (ECE), para medir el logro de los aprendizajes de los estudiantes en las capacidades de comprensión lectora y matemáticas, en las instituciones educativas (públicas y privadas) de todo el país, e informar de los resultados a la sociedad en general. Esta información permite al sistema conocer la evolución de los aprendizajes evaluados de forma comparativa a lo largo de los años.

Diversos informes concluyen que las brechas de aprendizaje entre los estudiantes de las zonas rurales respecto de sus pares de las zonas urbanas todavía subsisten (CNE, 2016).

En la tabla 3 se constata estas disparidades en los logros de aprendizaje que la ECE ha ido mostrando, según el área geográfica, sucesivamente:

Tabla 3. Resultados de las ECE 2016 región Cusco en el segundo grado de primaria en IIEE urbanas y rurales.

\begin{tabular}{|l|c|c|c|c|c|c|c|c|c|c|c|c|}
\hline \multirow{2}{*}{$\begin{array}{l}\text { Evaluaciones } \\
\text { censales }\end{array}$} & \multicolumn{4}{|c|}{ Uectura } & \multicolumn{5}{c|}{ Matemática } \\
\cline { 2 - 14 } & EI & EP & S & EI & EP & S & EI & EP & S & EI & EP & S \\
\hline ECE 20I6 & $3, I \%$ & $45,4 \%$ & $5 I, 5 \%$ & $16,4 \%$ & $63,4 \%$ & $20,1 \%$ & $19,0 \%$ & $40,2 \%$ & $40,8 \%$ & $47,6 \%$ & $35,8 \%$ & $16,6 \%$ \\
\hline ECE 20I5 & $3,0 \%$ & $40,3 \%$ & $56,7 \%$ & $20,8 \%$ & $64, I \%$ & I5,I\% & $22,7 \%$ & $45,0 \%$ & $32,3 \%$ & $56,6 \%$ & $35,2 \%$ & $8,2 \%$ \\
\hline ECE 20I4 & $9,3 \%$ & $45,6 \%$ & $45,1 \%$ & $42,2 \%$ & $49,1 \%$ & $8,7 \%$ & $32,4 \%$ & $37,6 \%$ & $30,0 \%$ & $69,5 \%$ & $23,4 \%$ & $7,0 \%$ \\
\hline
\end{tabular}

Fuente: Elaboración propia con datos de la Unidad de Medición de la Calidad (UMC). Nota. EI: en inicio, EP: en proceso, S: satisfactorio. 
La tabla 3 muestra que, en comprensión lectora, en las tres últimas evaluaciones censales, el porcentaje de estudiantes de la zona rural que se ubican en el nivel satisfactorio se ha venido incrementando de 8,7\% (ECE 20I4) a 20,I\% (ECE 20I6). En cuanto a las capacidades matemáticas este incremento fue de 7,0\% (ECE 20I4) a I6,6\% (ECE 2016). Se trata de un progreso importante en la educación primaria de las zonas rurales; empero, si se toma en cuenta los resultados de los estudiantes de las instituciones educativas de las zonas urbanas, se comprueba que estos logros todavía son insuficientes.

Al respecto, el Consejo Nacional de Educación (CNE) manifiesta que:

A pesar de que, en los últimos años, el sector educación ha empezado a atender algunos de los problemas específicos de la educación rural, la brecha en resultados de aprendizaje entre la población urbana y rural no disminuye, pues las mejoras en el medio urbano van a un ritmo más acelerado que en el rural. (p. 7).

Todas las estrategias implementadas por el Estado para revertir esta situación de inequidad educativa (evaluaciones censales, acompañamiento y monitoreo, apoyo alimentario, salud escolar, entre otros) han sido importantes; sin embargo, resultan todavía insuficientes, por lo que es necesario seguir repensando el diseño de estas acciones que contribuyan, efectivamente, a la construcción de un modelo pertinente de gestión para las IIEEUM.

\section{Metodología}

El estudio realizado adopta un enfoque cuantitativo, de tipo descriptivo, por cuanto busca describir la progresión de los aprendizajes de los estudiantes del segundo grado de primaria de las IIEEUM de la región Cusco, a partir de los resultados obtenidos en el nivel satisfactorio, en comprensión lectora y matemática, en las evaluaciones censales de los años 2014, 2015 y 2016.

Se trata de una investigación exploratoria, pues no se cuenta con estudios previos sobre logros de aprendizaje en las IIEEUM en la región Cusco. Por lo mismo, a partir de estos hallazgos, se busca crear una base inicial de conocimiento que sirva de inspiración para 
futuras investigaciones y para el diseño de proyectos educativos que contribuyan en el mejoramiento de los aprendizajes en los niños y niñas de estas instituciones educativas.

Asimismo, de acuerdo con la clasificación de los diseños de investigación planteado por Hernández, Fernández y Baptista (20I4), este estudio adopta un diseño longitudinal de tendencia, por cuanto analiza la progresión de los aprendizajes de los estudiantes del segundo grado de primaria de las IIEEUM, de las zonas rurales, a partir de los resultados obtenidos en el nivel satisfactorio, en las evaluaciones censales de los años 2014, 2015 y 2016.

La población y muestra está representada por aquellos estudiantes del segundo grado de primaria de las IIEEUM de la región Cusco, que participaron en las ECE y que alcanzaron el nivel satisfactorio en comprensión lectora y matemáticas. Esto es preciso aclararlo, por cuanto el marco muestral de la ECE excluye a los estudiantes que asisten a una institución educativa que atiende a menos de cinco estudiantes. Dicha exclusión responde a motivos técnicos y logísticos, entre otros.

El proceso de validación de las pruebas ha seguido una tabla de especificaciones que se encuentra en el Marco de Trabajo de la ECE (Minedu, 2009). En dicho documento se explica el enfoque de cada área; los objetivos y objetos (variables) de la evaluación; las características de la población evaluada; las competencias, capacidades e indicadores evaluados; el modelo y los contextos de evaluación, y, en el caso específico de Comunicación, los tipos y géneros textuales utilizados en la evaluación. Asimismo, en las tablas de especificaciones se precisan la cantidad y la proporción asignada a cada capacidad, indicador y descriptor por evaluar. En el caso de Comunicación, también se precisan la cantidad y la proporción de los diferentes tipos y géneros textuales.

Las pruebas fueron analizadas aplicando el modelo Rasch, cuyos indicadores se presentan en la tabla 4: 
Tabla 4. Propiedades psicométricas de la ECE.

\begin{tabular}{|l|r|r|}
\hline Indicador & Prueba & \multicolumn{1}{|c|}{ Lectura } \\
\hline Confiabilidad & 0,80 & 0,85 \\
\hline Ajuste al modelo: & & \\
Infit & $0,84-\mathrm{I}, 17$ & $0,83-\mathrm{I}, \mathrm{I0}$ \\
Outfit & $0,5 \mathrm{I}-\mathrm{I}, 29$ & $0,7 \mathrm{I}-\mathrm{I}, 47$ \\
\hline Unidimensionalidad: & & \\
Primer autovalor & 2,0 & $\mathrm{I}, 8$ \\
Varianza del primer autovalor & $3,5 \%$ & $3,3 \%$ \\
\hline
\end{tabular}

Fuente: Reporte técnico de la evaluación censal de estudiantes (ECE 2015) segundo y cuarto; (EIB) de primaria, segundo de secundaria. (2015).

Para efectos del presente estudio se tomó la medida promedio, que viene a ser el promedio aritmético de los puntajes calculados mediante el modelo Rasch, el cual representa las habilidades logradas por los estudiantes de un determinado grupo o estrato (DRE, UGEL, gestión y áreas de la institución educativa, entre otros).

Las pruebas establecen niveles de logro, que son las descripciones de los conocimientos y habilidades que se espera demuestren los estudiantes en las pruebas aplicadas en la ECE. Con ello, los estudiantes pueden ubicarse en alguno de los niveles según su desempeño y el grado en que fueron evaluados. Estos niveles se muestran en el cuadro I:

Cuadro I. Niveles de aprendizaje establecidos en la ECE.

\begin{tabular}{|c|c|c|}
\hline EN INICIO & EN PROCESO & SATISFACTORIO \\
\hline $\begin{array}{l}\text { Medida promedio } \\
\text { menor a } 458\end{array}$ & $\begin{array}{l}\text { Medida promedio } \\
\text { entre } 458 \text { y } 583\end{array}$ & $\begin{array}{l}\text { Medida promedio } \\
\text { mayor a } 583\end{array}$ \\
\hline $\begin{array}{l}\text { El estudiante no logró } \\
\text { los aprendizajes espe- } \\
\text { rados para el III ciclo. } \\
\text { Solo logra realizar tareas } \\
\text { poco exigentes respecto } \\
\text { de lo que se espera para } \\
\text { este ciclo. }\end{array}$ & $\begin{array}{l}\text { El estudiante solo logró } \\
\text { parcialmente los apre- } \\
\text { ndizajes esperados al } \\
\text { finalizar el III ciclo. Se } \\
\text { encuentra en camino de } \\
\text { lograrlo, pero aún tiene } \\
\text { dificultades. }\end{array}$ & $\begin{array}{l}\text { El estudiante logró los } \\
\text { aprendizajes esperados } \\
\text { para el III ciclo y está } \\
\text { preparado para afrontar } \\
\text { los retos de aprendizaje } \\
\text { del ciclo siguiente. }\end{array}$ \\
\hline
\end{tabular}

Fuente: Elaboración propia con datos del Reporte técnico de la evaluación censal de estudiantes (ECE 2015) segundo y cuarto (EIB) de primaria, segundo de secundaria. (2015). 
Estos niveles de logro son inclusivos, vale decir, el estudiante que logre ubicarse en el nivel Satisfactorio (NS), además de lograr los aprendizajes correspondientes a este, también puede demostrar aprendizajes que competen a los otros dos niveles.

Todos los datos utilizados para la presente investigación constituyen parte de la Muestra de Control de la ECE, que corresponden a las tres últimas evaluaciones censales correspondientes a los años 20I4, 2015 y 2016, y que pertenecen a las IIEEUM de la región Cusco que participaron de dichas evaluaciones, información que fue proporcionada por el Área de Gestión Institucional de la Dirección Regional de Educación Cusco.

\section{Resultados}

Los aspectos metodológicos utilizados en la investigación permiten interpretar los resultados de aprendizaje obtenidos por los estudiantes del segundo grado de primaria de las IIEEUM de la región Cusco. Se tomó como referencia el nivel Satisfactorio (NS) en comprensión lectora y capacidades matemáticas, por cuanto la finalidad del sistema educativo peruano y de la sociedad en su conjunto es que todos los niños y niñas alcancen mejores aprendizajes con calidad y equidad. En ese entender, los resultados que a continuación se muestran responden a los objetivos específicos planteados para la presente investigación.

Objetivo I. Describir, en el nivel de cada UGEL, el porcentaje de estudiantes del segundo grado de primaria, de las IIEEUM, que logra el nivel Satisfactorio en comprensión lectora y capacidades matemáticas, en las ECE 2014, 2015 y 2016.

La tabla 5 presenta, de manera general, el porcentaje de estudiantes de las IIEEUM que alcanzaron el NS en comprensión lectora, en las tres últimas evaluaciones censales aplicadas por el Minedu, pudiéndose advertir que los progresos en las tres últimas evaluaciones censales muestran una relativa mejoría en el NS. 


\begin{tabular}{|c|c|c|c|c|c|c|c|c|c|c|c|c|c|c|c|c|}
\hline & $\alpha^{\circ}$ & $\left|\begin{array}{l}2 \\
0 \\
0 \\
\vec{v}\end{array}\right|$ & $\begin{array}{l}2 \\
0 \\
0 \\
0 \\
-\end{array} \mid$ & 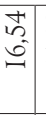 & 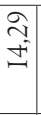 & 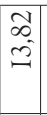 & $\begin{array}{l}\vec{O} \\
\stackrel{⿹}{0}\end{array}$ & 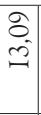 & $\stackrel{\widetilde{\Omega}}{\Xi}$ & $\begin{array}{l}\vec{b} \\
-\end{array}$ & $\begin{array}{l}0 \\
0 \\
0\end{array}$ & $\mid \begin{array}{l}0 \\
i \\
i n\end{array}$ & 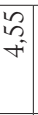 & $\left|\begin{array}{l|}8 \\
i \\
i\end{array}\right|$ & 8 & \\
\hline & $\bar{z}$ & tr & $\stackrel{\infty}{\infty}$ & $\tilde{N}$ & থ & F & $\ddot{m}$ & $\stackrel{2}{12}$ & $N$ & $m$ & 0 & 0 & $\Xi$ & + & 0 & 今ે \\
\hline 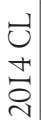 & $1-$ & 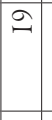 & ڤે & $\tilde{\Xi}$ & $\begin{array}{l}\widetilde{3} \\
\sim\end{array}$ & 号 & $\stackrel{\circ}{\sim}$ & in & ֶెర & $\vec{m}$ & Әे & $\underset{\Xi}{\Xi}$ & $\underset{\sim}{\stackrel{I}{\sim}}$ & 离 & 0 & $\begin{array}{l}\tilde{\Omega} \\
\hat{\sim}\end{array}$ \\
\hline પ્ & $\begin{array}{l}\text { 界 } \\
0 \\
0\end{array}$ & 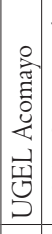 & 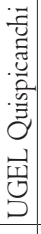 & 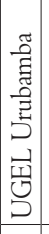 & 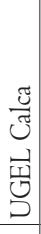 & 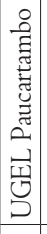 & 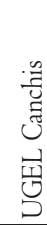 & 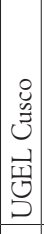 & 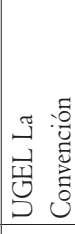 & 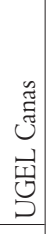 & 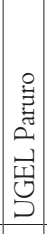 & 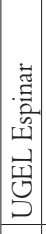 & 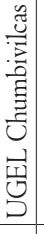 & 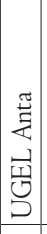 & 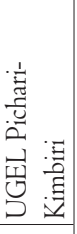 & 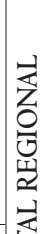 \\
\hline & $\stackrel{\circ}{Z}$ & - & $\sim$ & $\infty$ & + & in & 0 & $\Lambda$ & $\infty$ & $a$ & 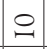 & $\Xi$ & $\simeq$ & $\Theta$ & \pm & 0 \\
\hline & & 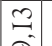 & & $\begin{array}{l}\infty \\
1 \\
-10\end{array}$ & & $\vec{\sigma}$ & $\hat{3}$ & $\because$ & 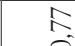 & $\hat{\sigma}$ & 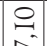 & \begin{tabular}{|l|}
0 \\
$n$ \\
3
\end{tabular} & $\because$ & 3 & & \\
\hline
\end{tabular}

:0

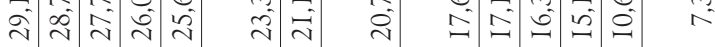

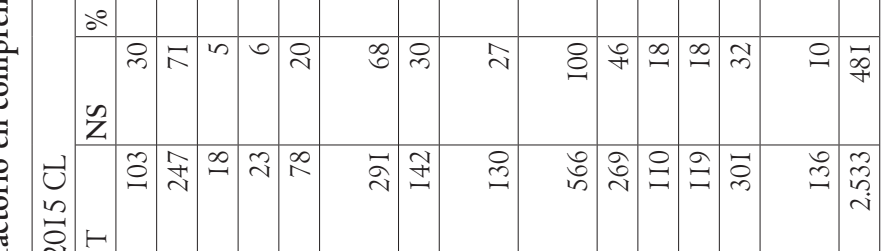

虫

.

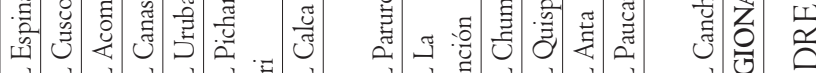

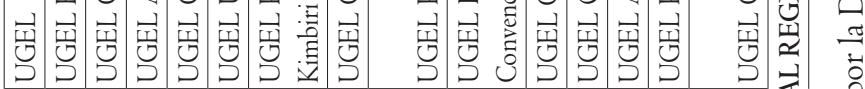

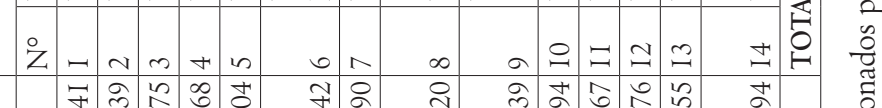

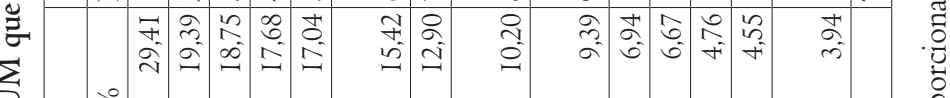

方

$\ddot{*}$

ว

นี

பี

常H

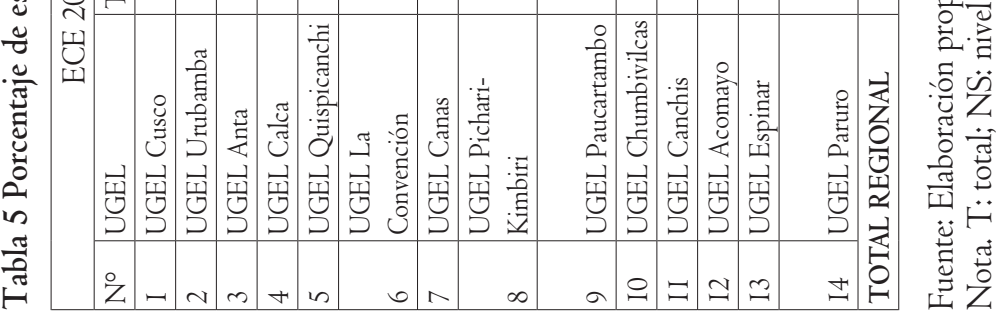


En la tabla 5 se aprecia que los estudiantes de las IIEEUM de la UGEL Cusco, en las ECE de 2014, 2015 y 2016 muestran interesante progreso en comprensión lectora. En la ECE 2014 se encontraban en el séptimo lugar, con $13,09 \%$ en el NS, en la ECE 2016 se sitúan en el nivel regional con 29,4I\%.

También, en el extremo inferior de la tabla 4 se puede advertir que el porcentaje de estudiantes que pertenecen a la UGEL Paruro, que en la ECE 2014 alcanzaban a un 6,06\% en el NS, en la ECE 2016 apenas llegaban a un $3,94 \%$, pese a que la matrícula escolar se había venido incrementando ligeramente. Asimismo, llama la atención el caso de la UGEL Espinar, que en la ECE 2014 contaba con 5, 26\% de estudiantes que alcanzaban el NS, en la ECE 2015 este porcentaje se incrementó a un 29,13\%; sin embargo, en la ECE 2016 disminuyó significativamente a un $4,55 \%$. No se cuenta con información sobre los factores que ocasionaron este notable descenso.

En lo que respecta a los progresos en capacidades matemáticas, la tabla 6 permite constatar que, en general, el porcentaje de estudiantes de las IIEEUM que lograron alcanzaron el NS en las tres últimas evaluaciones censales muestra un ligero incremento, a diferencia de los porcentajes obtenidos en comprensión lectora. 


\begin{tabular}{|c|c|c|c|c|c|c|c|c|c|c|c|c|c|c|c|c|c|}
\hline & $\partial^{\circ}$ & 足 & $\underset{ \pm}{ \pm}$ & $\stackrel{\infty}{+\infty}+$ & $\approx$ & సิ & సิ & ? & की & $\underset{\infty}{\stackrel{N}{N}}$ & 点 & जे & 요 & 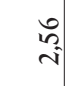 & $\stackrel{8}{\circ}$ & & \\
\hline & $\bar{Z}$ & in & $|\infty|$ & $\stackrel{\infty}{\sim}$ & $\stackrel{\sim}{\sim}$ & $\widetilde{\sim}$ & $\tilde{N}$ & $\Xi$ & $\approx$ & 으 & $a$ & $\infty$ & $N$ & - & 0 & 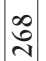 & \\
\hline $\mid$ & - & in & 윔 & 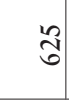 & ָี & $\tilde{\Theta}$ & $\tilde{\theta}$ & în & $\underset{7}{ \pm}$ & $\underset{\Xi}{\Xi}$ & $\stackrel{\sim}{二}$ & ڤే & Ә & సે & 0 & $\mid$ & \\
\hline પ્પ્પ & |⿹ & 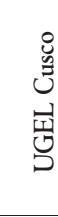 & 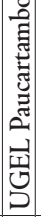 & 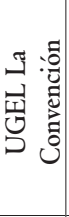 & 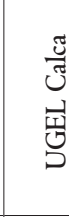 & 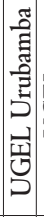 & 预 & 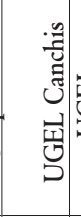 & 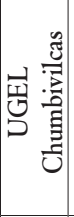 & 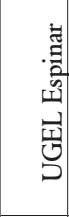 & 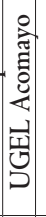 & 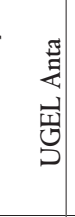 & 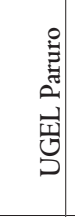 & : & 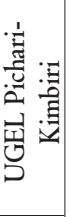 & 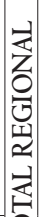 & \\
\hline & $\stackrel{8}{\text { ZZ }}$ & $\neg$ & $\sim$ & 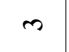 & + & 10 & 0 & $\sim$ & $\infty$ & $\sigma$ & 응 & $\Xi$ & $\simeq$ & 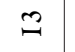 & $\Xi$ & 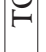 & \\
\hline & $\therefore$ & ? & 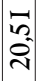 & $\begin{array}{l}\hat{N} \\
\stackrel{2}{a}\end{array}$ & $\stackrel{\tilde{I}}{\tilde{I}}$ & $\begin{array}{l}\infty \\
0 \\
i \\
\text { i }\end{array}$ & $\begin{array}{l}\widetilde{C} \\
\text { İ }\end{array}$ & 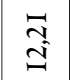 & $\begin{array}{l}\widetilde{\sigma} \\
\sigma\end{array}$ & $\begin{array}{l}0 \\
2 \\
\sigma^{2}\end{array}$ & స్. & $\overrightarrow{\vec{N}^{\prime}}$ & $\begin{array}{l}\text { in } \\
\text { in }\end{array}$ & $\begin{array}{l}\text { J } \\
\text { in }\end{array}$ & \begin{tabular}{l}
$\infty$ \\
\multirow{0}{0}{} \\
$\infty$
\end{tabular} & & \\
\hline & $\tilde{Z}$ & $\wedge$ & 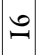 & ఉా & $\Xi$ & $\stackrel{\infty}{=}$ & $\cong$ & $\stackrel{\bullet}{\bullet}$ & $\stackrel{\infty}{\sim}$ & in & $\stackrel{\infty}{\sim}$ & $\widetilde{\sim}$ & $\varpi$ & 0 & $n$ & $\overrightarrow{\mathrm{N}}$ & \\
\hline $\begin{array}{l}\sum_{2} \\
\vdots \\
a\end{array}$ & $\mapsto$ & $\tilde{\sim}$ & $\stackrel{\infty}{\curvearrowright}$ & 竎 & $\varrho$ & $\mathbb{Z}$ & $\stackrel{\varrho}{\varrho}$ & $\widetilde{\Omega}$ & $\overrightarrow{\bar{\lambda}}$ & $\begin{array}{l}\stackrel{0}{\circ} \\
i n\end{array}$ & స్ల & $\stackrel{\infty}{\stackrel{\infty}{\sim}}$ & $\stackrel{\infty}{\sim}$ & $\stackrel{\varrho}{\Xi}$ & 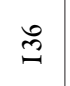 & $\mid \begin{array}{c}0 \\
\hat{n} \\
\text { î. }\end{array}$ & \\
\hline 夏 & 범 & 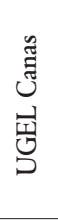 & 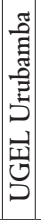 & 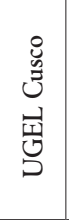 & 氙 & 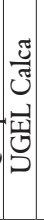 & 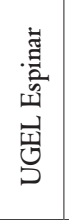 & 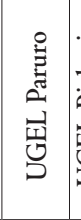 & 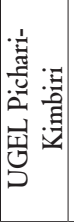 & 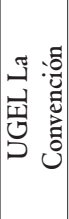 & | & 矛泀 & 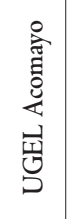 & 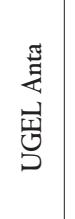 & 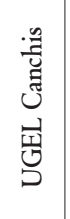 & 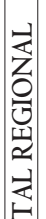 & 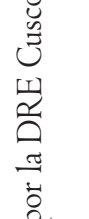 \\
\hline & $\stackrel{\circ}{\text { Z }}$ & $\neg$ & $\sim$ & $m$ & 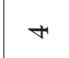 & in & 0 & $r$ & $\infty$ & $\sigma$ & 이 & $\Xi$ & 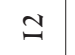 & $\cong$ & $\Xi$ & 의 & $\frac{\tilde{O}}{\tilde{C}}$ \\
\hline & $0^{\circ}$ & ָ̃ & 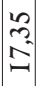 & $\begin{array}{l}2 \\
\stackrel{0}{5} \\
=\end{array}$ & $\underset{\mathbb{I}}{\mathbb{N}}$ & 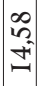 & 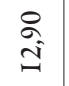 & $\begin{array}{l}R \\
\hat{0}\end{array}$ & $\begin{array}{l}3 \\
\tilde{0} \\
0\end{array}$ & బै & aे & ř & $\stackrel{2}{ \pm}$ & 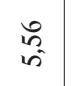 & $\stackrel{N}{\stackrel{N}{*}}$ & & 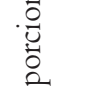 \\
\hline & $\bar{Z}$ & $\stackrel{\sim}{\sim}$ & 근 & กี & $\stackrel{\Xi}{\sim}$ & 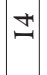 & 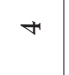 & 3 & $\widetilde{N}$ & $\sim$ & + & $\infty$ & $\curvearrowright$ & $\simeq$ & 0 & :ึ) & 总 \\
\hline 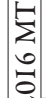 & - & $\begin{array}{l}n \\
\end{array}$ & $\mathscr{2}$ & 总 & త్ర & ঃ & $\varpi$ & ָ̃ & $\stackrel{\Xi}{\sim}$ & $\vec{\sim}$ & F & $\stackrel{2}{2}$ & 点 & $\stackrel{\bullet}{\sim}$ & $\widehat{\widehat{\beth}}$ & $\mid \begin{array}{l}\vec{\Xi} \\
\vec{N}\end{array}$ & 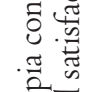 \\
\hline 넘 & : & 氙 & 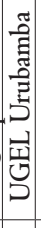 & 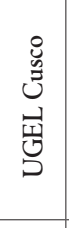 & 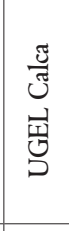 & 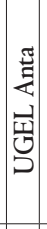 & 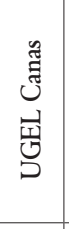 & 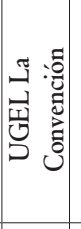 & 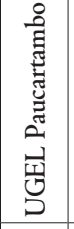 & 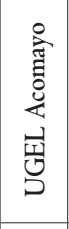 & 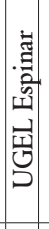 & 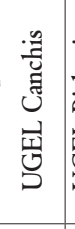 & 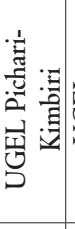 & 武 & 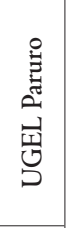 & 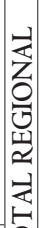 & 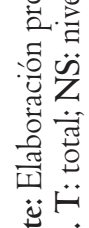 \\
\hline & 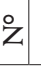 & - & $N$ & 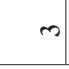 & H & 10 & 0 & 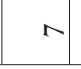 & $\infty$ & $\sigma$ & 은 & $\Xi$ & $\mathbb{Z}$ & $\tilde{\bullet}$ & \pm & $\stackrel{0}{\circ}$ & تُ \\
\hline
\end{tabular}


La tabla 6 muestra que, en la UGEL Quispicanchi, el porcentaje de estudiantes que alcanzaron el NS en capacidades matemáticas se ha incrementado en las tres últimas evaluaciones censales. En la ECE 2014 apenas alcanzaba a un I7,29\% de estudiantes; en la ECE 2016 este porcentaje de estudiantes en el NS llegó a un I9,26\%.

En el extremo inferior de la tabla 6 se puede constatar el caso de los estudiantes de las IIEEUM de la UGEL Paruro. A pesar de haber experimentado un repunte en la ECE 2015, con un I2,21\% de estudiantes en el NS, en la ECE 2016 este porcentaje se redujo a un $4,72 \%$.

De otro lado, en la UGEL de Chumbivilcas se aprecia una disminución notable en los porcentajes de estudiantes que alcanzaron el NS en las tres evaluaciones censales (ECE 20I4: 5,37\%; ECE 20I5: 8,2I y ECE 20I6: 5,56\%). No se muestran los resultados de la UGEL Pichari-Kimbiri en la ECE 20I4, por cuanto dicha UGEL no había sido creada.

Objetivo 2. Identificar las UGEL que tienen el mayor porcentaje de estudiantes del segundo grado de primaria de las IIEE UM en el nivel Satisfactorio, en comprensión lectora y capacidades matemáticas, en las ECE 20I4, 2015 y 2016.

Para realizar esta identificación se tomó la media aritmética a partir de los porcentajes de estudiantes que alcanzaron el NS en todas las UGEL, lo que permitió establecer dos grupos de referencia: el primero constituido por las UGEL que presentan relativamente los mejores porcentajes de estudiantes que alcanzan el NS (en el orden de I al 7), y el segundo integrado por el resto de las UGEL, en el cual el porcentaje de estudiantes que alcanzan el NS es mínimo, por debajo de la media aritmética.

En la tabla 7 se puede verificar las UGEL cuyos estudiantes, de las IIEEUM de su jurisdicción, presentan porcentajes mejores en el NS (parte sombreada de la tabla), en comprensión lectora. 


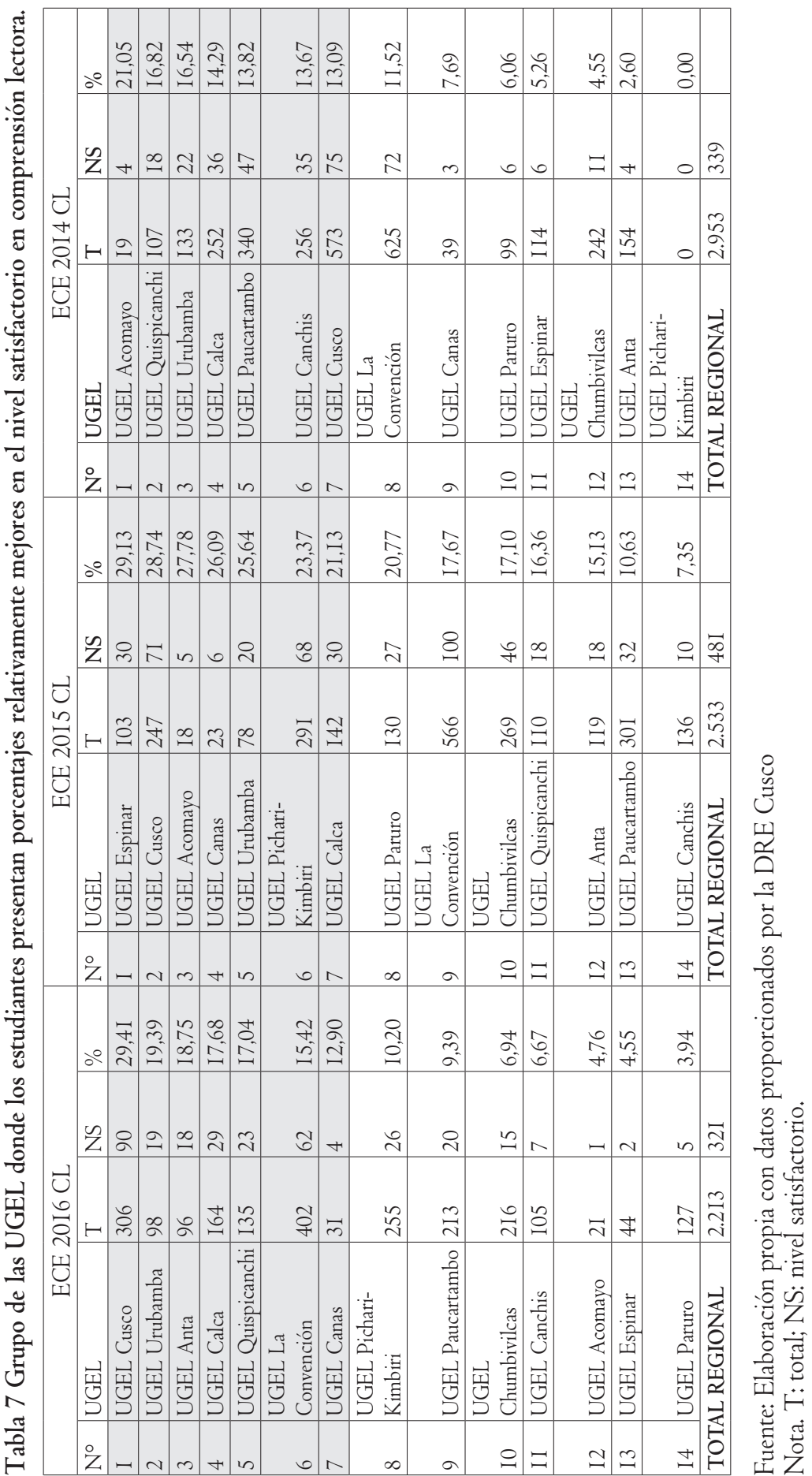


$\mathrm{Al}$ tomar como referencia de este análisis los resultados obtenidos por las UGEL en la ECE 20I4, y que se encontraban en el primer grupo (en el orden del I al 7), se aprecia que algunas de estas han disminuido en el porcentaje y pasan a ocupar el segundo grupo en la ECE 2016. Es el caso de la UGEL Acomayo. También hay casos de algunas UGEL que, habiéndose ubicado en el segundo grupo en la ECE 20I4, en la ECE 2016 logran incrementar el porcentaje de estudiantes en el NS y se posicionan en el primer grupo. La UGEL Canchis, que se situaba en el primer grupo de UGEL con mejores resultados en la ECE 20I4, deja este grupo y, finalmente, ocupa el onceavo lugar en la ECE 20I6. Es el mismo caso que presenta la UGEL Paucartambo.

En cuanto a las capacidades matemáticas, la tabla 8 presenta los resultados en el nivel de cada UGEL, en donde se aprecia, en ambos grupos, una clara disminución en los porcentajes de estudiantes que alcanzan el NS. 


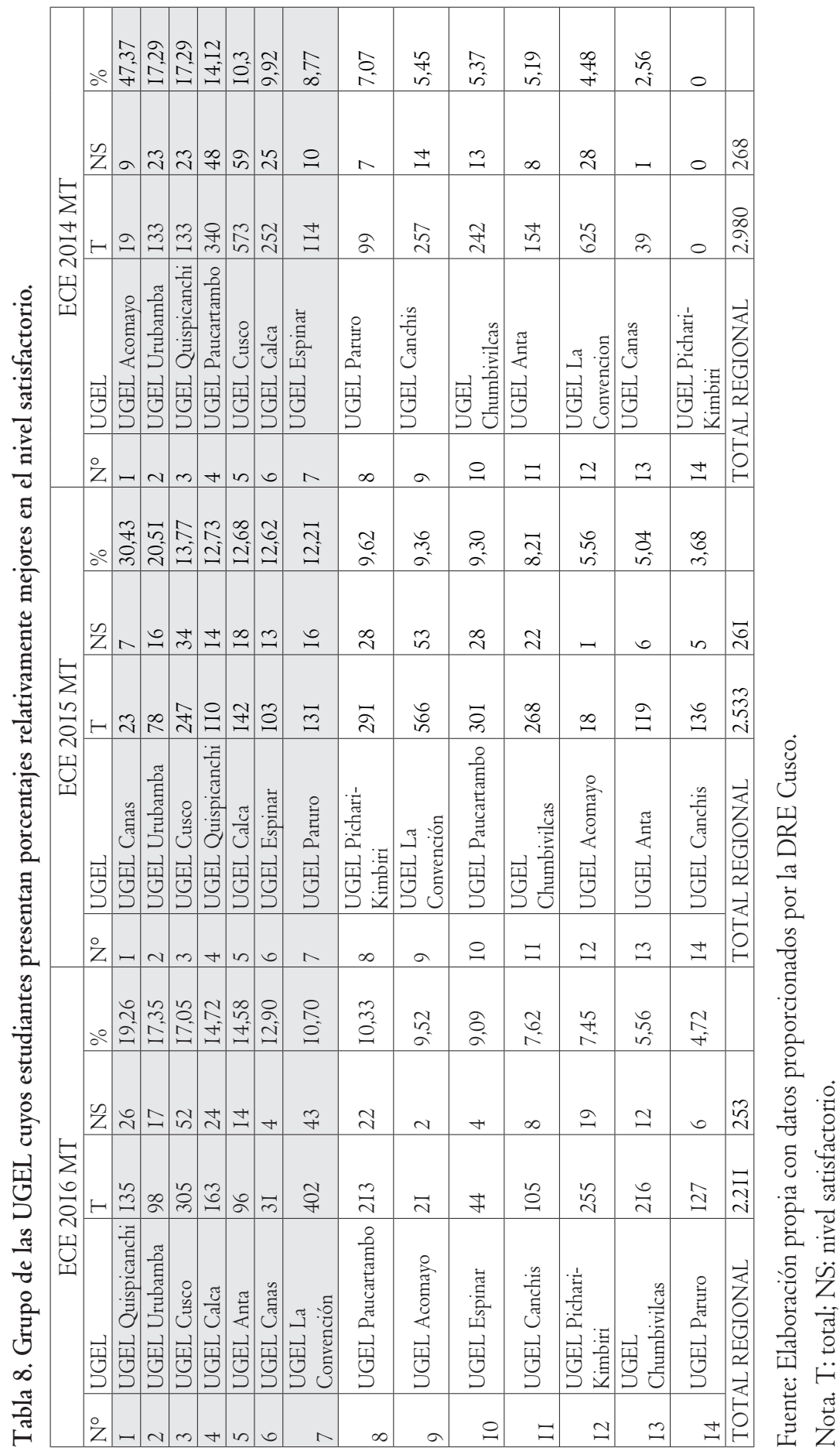


En la tabla 8 se constata el caso de la UGEL Acomayo, que en la ECE 2014 se situaba en el primer lugar con 47,37\% de estudiantes de las IIEEUM en el NS en matemática; a partir de la ECE 2015 sale de este primer grupo y se ubica en el segundo grupo de UGEL que presenta porcentajes mínimos en matemática. Es el mismo caso de la UGEL de Paucartambo.

La UGEL Canas, la UGEL La Convención y la UGEL Anta, que en la ECE 20I4 se encontraban en el segundo grupo de UGEL con resultados mínimos, en la ECE 2016 logran situarse en el primer grupo.

Objetivo 3. Determinar el efecto del porcentaje de la totalidad de estudiantes del segundo grado de primaria de las IIEEUM que alcanzaron el nivel Satisfactorio en las ECE 20I4, 2015 y 2016, en el porcentaje general total de la región Cusco.

La tabla 9 permite verificar el porcentaje total de estudiantes de las IIEEUM de la región Cusco que alcanzaron el NS en comprensión lectora y capacidades matemáticas, y su efecto en el porcentaje total regional.

Tabla 9. Porcentaje de estudiantes de las IIEEUM que aportan en el porcentaje regional en las ECE 20I4, 2015 y 20I6, en el nivel satisfactorio.

\begin{tabular}{|c|l|l|l|l|l|l|l|l|l|}
\hline \multirow{2}{*}{$\begin{array}{c}\text { Capacidades } \\
\text { evaluadas }\end{array}$} & \multicolumn{3}{|c|}{ ECE 20I6 } & \multicolumn{3}{c|}{ ECE 20I5 } & \multicolumn{3}{c|}{ ECE 20I4 } \\
\cline { 2 - 10 } & $\begin{array}{l}\text { Total } \\
\text { UM }+ \\
\text { PC } \\
\text { Región } \\
\text { Cusco }\end{array}$ & $\begin{array}{l}\text { Total, } \\
\text { NS } \\
\text { UM } \\
\text { Región } \\
\text { Cusco }\end{array}$ & $\%$ & $\begin{array}{l}\text { Total } \\
\text { NS } \\
\text { UM }+ \\
\text { PC } \\
\text { Región } \\
\text { Cusco }\end{array}$ & $\begin{array}{l}\text { Total, } \\
\text { NS } \\
\text { UM } \\
\text { Región } \\
\text { Cusco }\end{array}$ & $\begin{array}{l}\text { NS } \\
\text { UM }+ \\
\text { PC } \\
\text { Región } \\
\text { Cusco }\end{array}$ & $\begin{array}{l}\text { Total, } \\
\text { NS } \\
\text { UM } \\
\text { Región } \\
\text { Cusco }\end{array}$ & $\%$ \\
\hline $\begin{array}{l}\text { Comprensión } \\
\text { Lectora }\end{array}$ & I0.046 & $32 \mathrm{I}$ & $3,30 \%$ & 10.248 & $48 \mathrm{I}$ & $4,92 \%$ & 7.522 & 339 & $4,72 \%$ \\
\hline $\begin{array}{l}\text { Capacidades } \\
\text { Matemáticas }\end{array}$ & $8.10 \mathrm{I}$ & 253 & $3,22 \%$ & 6.168 & $26 \mathrm{I}$ & $4,42 \%$ & 5.433 & 282 & $5,47 \%$ \\
\hline
\end{tabular}

Fuente: Elaboración propia con datos obtenidos por la DRE Cusco.

Nota. NS: nivel satisfactorio; UM: unidocente multigrado; PC: polidocente completo.

Para demostrar la contribución del porcentaje de estudiantes del segundo grado de las IIEEUM que alcanzaron el NS en las ECE 20I4, 2015 y 20I6, en el porcentaje general regional, previamente 
se hizo una sumatoria total de la cantidad estudiantes del segundo grado de las IIEEUM de cada UGEL que alcanzaron el NS en comprensión lectora y capacidades matemáticas y, enseguida, se realizó otra sumatoria de la cantidad de estudiantes de las instituciones educativas polidocentes completas (IIEEPC) que alcanzaron el NS en comprensión lectora y capacidades matemáticas. Ambas cantidades se sumaron y, de este resultado total, se obtuvo la diferencia con la cantidad de estudiantes de las IIEEUM del nivel regional.

En la ECE 20I4, en el nivel de la región Cusco, 7.522 estudiantes (entre IIEEUM e IIEEPC) alcanzaron el NS en comprensión lectora. De este total, solo 399 estudiantes (que representa un 4,72\%) pertenecen a las IIEEUM. Regionalmente se aprecia un ligero incremento (4,92\%); sin embargo, en la ECE 2016 se presenta una disminución notoria en este nivel, donde el porcentaje apenas alcanza a un $3,30 \%$ del total regional.

En cuanto a las capacidades matemáticas, en la ECE 20I4, en el total regional, se tuvo 5.433 estudiantes que lograron el NS; de estos únicamente 282 estudiantes (que representa el 5,47\%) de las IIEEUM alcanzaron dicho nivel. Si se observa el porcentaje de estudiantes de IIEEUM que se ubicaron en el NS en capacidades matemáticas, en la ECE 2016 se aprecia una disminución significativa que alcanza a un $3,22 \%$.

\section{Conclusiones}

I. Existe un persistente rezago en los aprendizajes de los niños y niñas de las IIEEUM de la región Cusco, según se observa en los resultados obtenidos en las tres últimas evaluaciones censales. Un mínimo porcentaje de la totalidad de estos alcanzan el NS en comprensión lectora. En el caso de las capacidades matemáticas, este porcentaje es todavía mucho menor, lo que confirma la existencia de una gran brecha de aprendizajes entre los estudiantes de las IIEEUM y sus pares que estudian en las IIEEPC. Estos resultados podrían explicarse a partir de las características y condiciones que presentan las IIEEUM (Ames, 2004), del tiempo efectivo para el aprendizaje del que disponen 
los profesores-directores, de si el profesor está debidamente preparado para desempeñarse mejor en un aula heterogénea (Anijovich, 20I4), si maneja el idioma materno de los niños y niñas, del desarrollo de los procesos pedagógicos, de si el diseño de la evaluación responde o no a las características de la realidad lingüística y cultural de los estudiantes, entre otras condiciones. A pesar de la ratio (proporción) profesor-estudiantes que es I a I3 (Escale, 2017) y de los vínculos socioemocionales que se promueven en el aula (Berger, Álamos \& Milicic, 2016), estos dos factores parecieran no influir en la mejora de los aprendizajes de los niños y niñas de las IIEEUM.

2. Son tres las UGEL (UGEL Calca, UGEL Urubamba y UGEL Cusco) que en las tres últimas evaluaciones censales se han mantenido de manera sostenida en el grupo que presenta los mejores porcentajes de estudiantes en el NS. No se cuenta con información detallada acerca de los factores que estarían favoreciendo estos logros.

3. En relación al efecto del porcentaje de total de estudiantes del segundo grado de primaria de las IIEEUM, región Cusco, que alcanzan el NS en el porcentaje regional, se puede afirmar que este efecto es muy reducido. El porcentaje de estudiantes de las IIEEUM que alcanza el NS en capacidades matemáticas es mínimo, a diferencia de sus pares que estudian en las IIEEPC. Además, dichos porcentajes de estudiantes que alcanzan el NS disminuyen notoriamente desde la ECE 2015 a la ECE 20I6, sobre todo en los aprendizajes de las capacidades matemáticas.

\section{Sugerencias}

Los resultados y las conclusiones extraídas del estudio permiten ratificar la persistencia de los problemas de inequidad educativa en las zonas rurales de la región Cusco, en especial en las IIEEUM. En consecuencia, para iniciar una mejora significativa de los aprendizajes de los niños y niñas de las zonas rurales se sugiere, entre otras acciones:

I. Diseñar e implementar un Modelo de Institución Educativa Unidocente Multigrado para garantizar un mejor servicio educativo 
que responda a las características actuales del contexto rural (diversidad, dinamismo, movilidad, entre otras) y que permita asegurar una mejora sostenida en los logros de aprendizaje de los estudiantes de las IIEEUM, así como revertir los altos índices de repitencia, extraedad y deserción escolar, según establece el derecho a la educación en la legislación educativa peruana.

2. La implementación de este modelo posibilitaría una intervención sectorial e intersectorial más eficaz, mejor coordinada, mediante los diversos programas que brinda el Minedu y de aquellos otros relacionados con la salud y la alimentación escolar.

3. Las ayudas que reciben las IIEUM desde otras instancias del Estado (municipalidades distritales, provinciales y de los mismos gobiernos regionales) para el mejoramiento de la infraestructura y equipamiento escolar (recursos y materiales educativos), deberían considerar necesariamente un componente que atienda el desarrollo de capacidades de los docentes que laboran en dichas instituciones educativas.

4. La formación continua de los docentes de las IIEEUM debería tener como foco principal la atención a la heterogeneidad de los estudiantes en las aulas, tal como establece el Marco de Buen Desempeño Docente y la Evaluación del Desempeño Docente.

5. Sería conveniente insistir con la estrategia de integrar algunas IIEEUM, analizando las condiciones geográficas de acceso de los niños y niñas a estas. Esta acción favorecería una mejor atención de los diversos programas de intervención del Estado (mediante una articulación intersectorial), a la par que permitiría que los profesores que trabajan aisladamente tengan más oportunidades de integrar una comunidad de aprendizaje profesional.

\section{Referencias bibliográficas}

Ames, P. (2004). Las escuelas multigrado en el contexto educativo actual: desafíos y posibilidades. Recuperado de https://www.researchgate. net/publication/39724196_Las_escuelas_multigrado_en_el_ contexto_educativo_actual_desafios_y_posibilidades

Anijovich, R. (20I4). Gestionar una escuela con aulas beterogéneas. Enseñar y aprender en la diversidad. Buenos Aires, Editorial Paidós. 
Berger, C., Álamos, P. y Milicic, N. (2016). El rol de los docentes en el aprendizaje socioemocional de sus estudiantes: la perspectiva del apego escolar, en Manzi, J. y García M. R. (Ed.), Abriendo las puertas del aula: Transformaciones de las prácticas docentes (pp. 383-4II). Santiago de Chile: Francisco Zabaleta CEPPE-UC.

Consejo Nacional de Educación. (2016). Líneas Prioritarias de Política Educativa al 2021, Año del Bicentenario. Acelerar el cambio educativo para el bienestar de todos y el desarrollo del país. Recuperado de http:/ /www.cne.gob.pe/uploads/ home/documentoprioridades I60I I6.pdf

ESCALE (2018). Tamaño promedio de clase, primaria (número de alumnos). Recuperado de http://escale.Minedu.gob.pe/

Fumagalli, L. (2015). La mejora de los resultados del aprendizaje escolar: qué hacer desde las políticas curriculares, en Poggi, M. (Coord.), Mejorar los aprendizajes en la educación obligatoria. Políticas y actores (pp. I5-44). Recuperado de http://unesdoc.unesco.org/ images/0023/002349/234977s.pdf

Guadalupe, C., León, J., Rodríguez, J. y Vargas, S. (2017). Estado de la educación en el Perú. Análisis y perspectivas de la educación básica. Lima: Impresiones y Ediciones Arteta E.I.R.L.

Instituto peruano de evaluación, acreditación y certificación de la calidad de la Educación Básica (IPEBA) (20I I). Ruralidad y escuela. Apuntes para la acreditación de instituciones educativas en áreas rurales. Recuperado de https:/ / www.sineace.gob.pe/wp-content/uploads/2015/06/Ruralidad-yEscuela.pdf

Latapí, P. (2009). El derecho a la educación: su alcance, exigibilidad y relevancia para la política educativa. Revista Mexicana de Investigación Educativa, 14(40), 255-287. Recuperado de http://www.redalyc.org/articulo. oa?id= 14004012

León, J. y Collahua, Y. (2016). El efecto del nivel socioeconómico en el rendimiento de los estudiantes peruanos: un balance de los últimos I5 años. En GRADE (Ed) Investigación para el desarrollo en el Perú. Once balances (pp. I09-I4I). Recuperado de https://www.grade.org.pe/wp content/ uploads/LIBROGRADE_DESARROLLO35.pdf

Ministerio de Educación del Perú. (2005). Ley General de Educación Ley No 28.004. Lima: MV FENIX E.I.R.L.

Ministerio de Educación del Perú. (2007). Proyecto Educativo Nacional al 202 l. La educación que queremos para el Perú. Recuperado de http://www.Minedu. gob.pe/DeInteres/xtras/PEN-202I.pdf 
Ministerio de Educación del Perú. (2009). Evaluación Censal de Estudiantes (ECE). Segundo grado de primaria y cuarto grado de primaria de IE EIB. Marco de trabajo. Recuperado de http://www2.Minedu.gob.pe/umc/ece/Marco_de_ Trabajo_ECE.pdf

Ministerio de Educación del Perú. (2012). Marco de Buen Desempeño Docente. Recuperado de http://www.Minedu.gob.pe/pdf/ed/marco-de-buendesempeno-docente.pdf

Ministerio de Educación del Perú. (2015). Reporte técnico de la evaluación censal de estudiantes (ECE 2015) segundo y cuarto (EIB) de primaria, segundo de secundaria. Recuperado de http://umc.Minedu.gob.pe/wp-content/ uploads/2016/03/Reporte-Tecnico-ECE2015.pdf

Ministerio de Educación del Perú. (2017). Informe de resultados de la Evaluación Censal de Estudiantes 2007-2015. Recuperado de http://umc.Minedu. gob.pe/wpcontent/uploads/2017/07/Informe-final_ECE-200720I5-vfinal.pdf

Miranda, L. (2008). Factores asociados el rendimiento escolar y sus implicancias para la política educativa del Perú, en Benavides M. (Ed.), Análisis de programas, procesos y resultados educativos en el Perú. Contribuciones empíricas para el debate. GRADE (pp. II-39). Recuperado de http://www.grade.org. $\mathrm{pe} / \mathrm{upload} / \mathrm{publicaciones/archivo/download/pubs/analisisO.pdf}$

Naciones Unidas. (1948). La Declaración Universal de Derechos Humanos. Recuperado de http://www.un.org/es/universal-declaration-human-rights/

Vargas, T. (2003). Escuelas multigrados: ¿cómo funcionan? Reflexión a partir de la experiencia evaluativa. Cuadernos, Unesco. Recuperado de http://unesdoc. unesco.org/images/0013/001374/137497so.pdf

Vigo, G. y Nakano, T. (2007). El derecho a la educación en el Perú. FLAPE. Recuperado de http://dide.Minedu.gob.pe/bitstream/ handle/I $23456789 / 53$ I / 299.\%20E1\%20derecho\%20 a\%201a\%20educaci\% C3\%B3n\%20en \%20Per\%C3\%BA. pdf?sequence $=\mathrm{I} \&$ is Allowed $=\mathrm{y}$ 